\title{
A Comparison of Colorimetric Assessment of Vaginal pH with Nugent Score for the Detection of Bacterial Vaginosis
}

\author{
Matthew K. Hoffman, ${ }^{1}$ Mrutyunjaya B. Bellad, ${ }^{2}$ Umesh S. Charantimath, ${ }^{2}$ \\ Avinash Kavi, ${ }^{2}$ Jyoti M. Nagmoti, ${ }^{2}$ Mahantesh B. Nagmoti, ${ }^{2}$ Ashalata A. Mallapur, ${ }^{3}$ \\ Geetanjali M. Katageri, ${ }^{3}$ Umesh Y. Ramadurg, ${ }^{3}$ Sheshidhar G. Bannale, ${ }^{3}$ \\ Amit P. Revankar, ${ }^{2}$ M. S. Ganachari, ${ }^{2}$ Richard J. Derman, ${ }^{4}$ and Shivaprasad S. Goudar ${ }^{2}$ \\ ${ }^{1}$ Department of Obstetrics \& Gynecology, Christiana Care Health System, Newark, DE, USA \\ ${ }^{2}$ KLE University Jawaharlal Nehru Medical College, Belgaum, Karnataka, India \\ ${ }^{3}$ S. Nijalingappa Medical College and HSK Hospital and Research Centre, Bagalkot, Karnataka, India \\ ${ }^{4}$ Thomas Jefferson University, Philadelphia, PA, USA \\ Correspondence should be addressed to Matthew K. Hoffman; mhoffman@christianacare.org
}

Received 6 October 2016; Revised 27 December 2016; Accepted 9 January 2017; Published 15 February 2017

Academic Editor: Bryan Larsen

Copyright ( 2017 Matthew K. Hoffman et al. This is an open access article distributed under the Creative Commons Attribution License, which permits unrestricted use, distribution, and reproduction in any medium, provided the original work is properly cited.

\begin{abstract}
Background. A Nugent score $>7$ has been defined as the gold standard for the diagnosis for bacterial vaginosis (BV), though it is resource intensive and impractical as point of care testing. We sought to determine if colorimetric assessment of vaginal $\mathrm{pH}$ can accurately predict the occurrence of BV. Methods. We performed a planned subanalysis of 1,216 pregnant women between $130 / 7$ and 19 6/7 weeks who underwent vaginal examination as part of a randomized controlled trial. Using a standardized technique, specimens were obtained for colorimetric assessment and two separate slides for Gram staining. These slides were subsequently evaluated by two independent blinded microbiologists for Nugent scoring. Results. Interrater reliability of the interpretation of the Nugent score was excellent (intraclass correlation-individual 0.93 (95 CI 0.92 to 0.94 ) and average 0.96 (95\% CI 0.95 to 0.97)). The sensitivity of an elevated $\mathrm{pH}>5$ for a Nugent score $>7$ was $21.9 \%$ while the specificity was $84.5 \%$. The positive predictive value in our population was $33.7 \%$ with a negative predictive value of $75.0 \%$. Conclusion. Though the Nugent score is internally accurate, the prediction of $\mathrm{BV}$ using vaginal $\mathrm{pH}$ alone has poor sensitivity and specificity.
\end{abstract}

\section{Background}

Bacterial vaginosis (BV) remains the most common form of vaginitis affecting women globally [1] and has been linked to several poor outcomes including preterm birth [2] and posthysterectomy infection [3]. Nonetheless, $29 \%$ of US women are noted to meet diagnostic criteria of BV, though the majority are clinically asymptomatic [4]. Recently, genetic microbiome studies have demonstrated that the occurrence of BV represents an absence of acid forming morphotypes of lactobacillus [5]. This lack of lactobacilli is frequently accompanied by an overgrowth of Gardnerella vaginalis forming an infected biofilm and creating a permissive environment for the overgrowth of numerous anaerobic Gram negative rods [6]. Nonetheless, the vaginal microbiome, as defined by Nugent scoring, is noted to vary dramatically by region of the world [7].

Several prior studies have suggested that vaginal $\mathrm{pH}$ alone may be an accurate marker for the detection of $\mathrm{BV}$ [8-10]. These high correlations have not been witnessed in other studies. It is thus important to establish the accuracy of vaginal $\mathrm{pH}$ to detect $\mathrm{BV}$ in an area where the resource intensive approaches to Gram stain and light microscopy may not be readily available. We thus sought to determine the 
ability of vaginal $\mathrm{pH}$ to detect $\mathrm{BV}$ as defined by the Nugent score amongst pregnant women in a low middle income setting in southern India.

\section{Methods}

Prior to the initiation of the study, Institutional Review Board approval was obtained from both participating institutions (Jawaharlal Nehru Medical College, Belgaum, India, and Christiana Care Health Services, Newark, Delaware). This trial was a planned substudy of a prospective individually randomized trial of pregnant women with an elevated vaginal $\mathrm{pH}(\geq 5.0)$ who would be treated with Clindamycin or placebo (ClinicalTrials.Gov NCT01800825, ICTR CTRI/2013/07/003799). Pregnant women between the gestational ages of $130 / 7$ and $196 / 7$ weeks were invited to participate in the trial. To be included women were to have a singleton gestation with no anomalies by history. Women were likewise excluded if they had a history of taking antibiotics in the last 14 days, had a history of vaginal bleeding in the last 3 days, had a symptomatic vaginal discharge, or were unable to consent (age $<18$ years with no provisions for family/husband consent). Consistent with the guidelines of the ethics review committee, before each examination, a woman provided consent in her local language under the direct observation of trained field staff.

Our primary outcome was the presence of BV as defined as a Nugent score of 7 or greater [11]. Though competing definitions for BV exist, such as Amsel's criteria, the Nugent scoring system was chosen as it has been acknowledged as the gold standard [11, 12].

Prior to field initiation, certified Auxiliary Nurse Midwives (ANMs) were trained in a standardized methodology for both obtaining slides for Gram stain and vaginal $\mathrm{pH}$ using a video and direct observation by a central team member. Using a standardized speculum exam, specimens were taken from the lateral vaginal sidewalls. Gram stains were obtained using 2 separate acrylic swabs and then immediately plated on a clean glass slide that was allowed to air dry. These slides were transported to a central reading area where they underwent Gram stain. Nugent score was then read by two independent pathologists in accordance with accepted techniques [11]. Women who had a Nugent score of 7 or greater were deemed to have bacterial vaginosis. In cases wherein the two pathologists disagreed and with one scoring below 7 , the higher score was chosen to define the presence or absence of bacterial vaginosis.

Vaginal $\mathrm{pH}$ was determined by directly placing a small portion of $\mathrm{pH}$ paper in the same location that the vaginal swab for the Gram stain was obtained from and it remained until being saturated with vaginal fluid. The $\mathrm{pH}$ was then evaluated after the $\mathrm{pH}$ paper had been allowed to dry after 60 seconds. $\mathrm{PH}$ paper was universally obtained from Micro-Essential Laboratories (Hydrion 345 S/R Dispenser; Brooklyn, NY) and is able to discriminate $\mathrm{pH}$ in 0.5 moles per liter increments between 3.0 and 5.5. These were recorded and read independently of the Gram stains.

Statistical analysis consisted of performing an intraclass correlation (two-way mixed effect model) between the two
TABLE 1: Demographics.

\begin{tabular}{lcc}
\hline Variable & & $95 \%$ CI \\
\hline Age in years & 23.7 & 22.7 to 24.7 \\
Gravidity & 2.5 & 1.9 to 3.1 \\
Parity & 1.4 & 1.3 to 1.5 \\
Height in cm & 151.2 & 150.6 to 151.8 \\
Weight in kg & 46.0 & 45.2 to 46.9 \\
Years in school & 8.8 & 8.5 to 9.2 \\
Respiratory disease & $1.57 \%$ & \\
Cardiac disease & $0.94 \%$ & \\
Diabetes & $0.31 \%$ & \\
Other diseases & $0.63 \%$ & \\
\hline
\end{tabular}

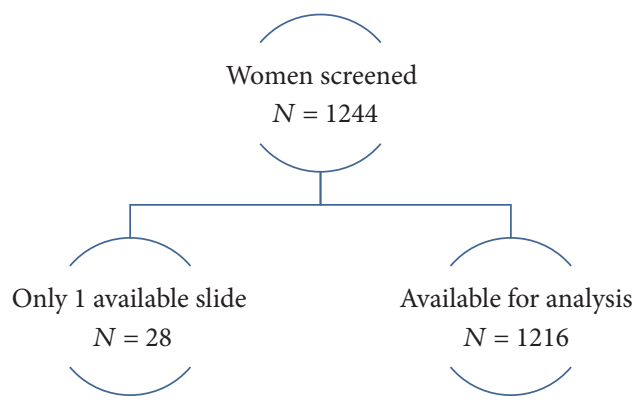

Figure 1

scoring microbiologists for both Nugent score and the occurrence of BV (Nugent score $\geq 7$ ). Comparison of the presence of BV defined by an elevated vaginal $\mathrm{pH}(\geq 5)$ versus BV defined by Nugent score was likewise compared using a pairwise correlation. Summary statistics were performed using simple univariate modelling. All statistical analysis were performed using STATA 14.0 (College Station TX).

\section{Results}

A total of 6,473 women underwent screening in the parent study; $26.7 \%(N=1,728)$ were found to have a vaginal $\mathrm{pH} \geq 5$. Of this cohort, a total of 1,244 women underwent screening with both a vaginal Gram stain and a vaginal $\mathrm{pH}$, of which $1,216(97.6 \%)$ met the entrance requirement (see Figure 1). Twenty-eight women were eliminated due to the lack of a second slide. Demographics of the participants are presented in Table 1. Our participants can best be summated as a young healthy but underweight cohort. This cohort is consistent with participants of our prior studies and the general population [13].

As determined by a Nugent score of 7 or greater, $17.4 \%$ of our participants had bacterial vaginosis. With reference to the Nugent score, there was a very high correlation between the two pathologists both between each other and the mean value. The intraclass correlation between raters was 0.929 (95\% CI 0.920 to 0.936 ) and between the average was 0.963 (95\% CI 0.959 to 0.967$)$ ( $P$ value $<0.001)$. This suggests that Nugent score is a highly accurate and consistent test. 


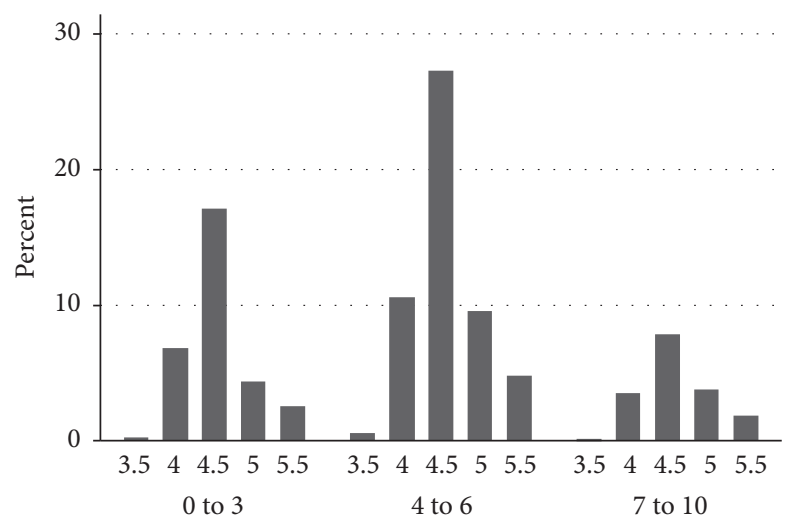

FIgURE 2: \% distribution by $\mathrm{pH}$ and Nugent grouping.

In contrast, vaginal $\mathrm{pH}$ fared poorly as a predictor of bacterial vaginosis defined as a Nugent score of 7 or greater. This is graphically illustrated in Figure 2, wherein the distribution of vaginal $\mathrm{pH}$ values is shown by Nugent groups ( 0 to 3,4 to 6 , and 7 to 10). In this graph one can see that the frequency of vaginal $\mathrm{pH}$ values appears to be normally distributed, not skewed with the higher $\mathrm{pH}$ values in the 7 to 10 group. The pairwise correlation between a $\mathrm{pH} \geq 5$ and an elevated Nugent score was 0.66 ( $P$ value of 0.021$)$. The sensitivity of an elevated $\mathrm{pH} \geq 5$ by Nugent score was $21.7 \%$ while the specificity was $84.1 \%$. The positive predictive value in our population was $31.1 \%$ with a negative predictive value of $76.4 \%$.

\section{Discussion}

The clinical diagnosis of bacterial vaginosis has long been made using Amsel's criteria (elevated vaginal $\mathrm{pH}$, presence of clue cells, milky discharge, and positive whiff test) or Nugent's criteria [14]. Through microbiotics, we have come to understand that bacterial vaginosis is a complex entity characterized largely as the absence of acid forming lactobacillus and a concomitant proliferation of other anaerobic bacteria [5]. This lack of acidity allows the proliferation of other organisms (microbial dysbiosis) which may be causative of underlying disease [15]. As lactobacilli play a key role in acidifying the vagina, it is not surprising that vaginal $\mathrm{pH}$ remains part of Amsel's criteria in making the clinical diagnosis of bacterial vaginosis.

Though we were able to demonstrate that an elevated vaginal $\mathrm{pH}$ is significantly associated with bacterial vaginosis as defined by Nugent's criteria, our measurement of vaginal $\mathrm{pH}$ had poor sensitivity and specificity compared to other publications. Amongst our participants, only $21.7 \%$ of women with an elevated vaginal $\mathrm{pH}$ (defined as vaginal $\mathrm{pH}$ or greater than or equal to 5.0.) had bacterial vaginosis. This finding is much more consistent with other reports that have found vaginal $\mathrm{pH}$ to be a relatively poor predictor of $\mathrm{BV}[8,10]$. This may in part be explained by the fact that vaginal microbiome of southern India may be markedly different than that in other countries [7].
Though broadly accepted as the gold standard, it is important to remember that the Nugent score reflects the relative ratios of large Gram positive rods (lactobacillus), small Gram variable rods, and curved Gram variable rods [11]. Whether these findings are diagnostic of BV and are associated with both disease and poor obstetrical outcomes in rural India where the microbiome may be different is unclear [7]. Further complicating the issue, recent genomic studies have demonstrated that "healthy" women who lack appreciable colonies of lactobacilli are relatively common $[16,17]$. These women appear to have other bacteria that are capable of acidifying the vagina, promoting microbial health. In such women, Nugent's criteria may not be an appropriate marker of the health of the vaginal microbiome.

Perhaps more important than how the diagnosis of bacterial vaginosis is derived is that an elevated vaginal $\mathrm{pH}$ represents an atypical vaginal microbiome free of acid forming organisms. Such an environment has been shown to provide permissive growth of pathological organisms like mycoplasma amongst others [18]. Several investigations have been able to link an elevated vaginal $\mathrm{pH}$ without either an elevated Nugent score or the other portions of Amsel's criteria with poor outcomes including preterm birth and preterm premature rupture of membranes [19-21]. It is for this reason that we will be exploring our obstetrical outcomes by vaginal $\mathrm{pH}$ in this unique cohort.

\section{Competing Interests}

None of the authors are aware of any financial relationships that would have influenced the conduct, analysis, or outcomes of this study.

\section{Acknowledgments}

This study was supported by the Thrasher Research Fund (Award no. 10326), Salt Lake City, Utah.

\section{References}

[1] L. O. Eckert, "Clinical practice. Acute vulvovaginitis," The New England Journal of Medicine, vol. 355, no. 12, pp. 1244-1252, 2006.

[2] M. A. Klebanoff, S. L. Hillier, R. P. Nugent et al., "Is bacterial vaginosis a stronger risk factor for preterm birth when it is diagnosed earlier in gestation?" American Journal of Obstetrics and Gynecology, vol. 192, no. 2, pp. 470-477, 2005.

[3] S. L. Hillier, R. P. Nugent, D. A. Eschenbach et al., "Association between bacterial vaginosis and preterm delivery of a low-birthweight infant. The Vaginal Infections and Prematurity Study Group," The New England Journal of Medicine, vol. 333, no. 26, pp. 1737-1742, 1995.

[4] J. E. Allsworth and J. F. Peipert, "Prevalence of bacterial vaginosis: 2001-2004 national health and nutrition examination survey data," Obstetrics \& Gynecology, vol. 109, no. 1, pp. 114-120, 2007.

[5] R. F. Lamont, J. D. Sobel, R. A. Akins et al., "The vaginal microbiome: new information about genital tract flora using molecular based techniques," BJOG: An International Journal of Obstetrics and Gynaecology, vol. 118, no. 5, pp. 533-549, 2011. 
[6] L. Hardy, V. Jespers, N. Dahchour et al., "Unravelling the bacterial vaginosis-associated biofilm: a multiplex Gardnerella Vaginalis and Atopobium vaginae fluorescence in situ hybridization assay using peptide nucleic acid probes," PLOS ONE, vol. 10, no. 8, Article ID e0136658, 2015.

[7] J. E. Tolosa, S. Chaithongwongwatthana, S. Daly et al., "The International Infections in Pregnancy (IIP) study: variations in the prevalence of bacterial vaginosis and distribution of morphotypes in vaginal smears among pregnant women," American Journal of Obstetrics and Gynecology, vol. 195, no. 5, pp. 11981204, 2006.

[8] Z. Mengistie, Y. Woldeamanuel, D. Asrat, and M. Yigeremu, "Comparison of clinical and gram stain diagnosis methods of bacterial vaginosis among pregnant women in Ethiopia," Journal of Clinical and Diagnostic Research, vol. 7, no. 12, pp. 2701-2703, 2013.

[9] J. S. Huppert, E. A. Hesse, M. C. Bernard, J. R. Bates, C. A. Gaydos, and J. A. Kahn, "Accuracy and trust of self-testing for bacterial vaginosis," Journal of Adolescent Health, vol. 51, no. 4, pp. 400-405, 2012.

[10] R. Hemalatha, B. A. Ramalaxmi, E. Swetha, N. Balakrishna, and P. Mastromarino, "Evaluation of vaginal $\mathrm{pH}$ for detection of bacterial vaginosis," Indian Journal of Medical Research, vol. 138, no. 3, pp. 354-359, 2013, http://www.pubmedcentral.nih.gov/artid=3818598\&tool=pmcentrez\&rendertype $=$ abstract.

[11] R. P. Nugent, M. A. Krohn, and S. L. Hillier, "Reliability of diagnosing bacterial vaginosis is improved by a standardized method of gram stain interpretation," Journal of Clinical Microbiology, vol. 29, no. 2, pp. 297-301, 1991.

[12] "Sexually transmitted diseases treatment guidelines, 2015," Morbidity and Mortality Weekly Report (MMWR), vol. 64, no. 3, pp. 1-137, 2015, https://www.cdc.gov/std/tg2015/tg-2015-print.pdf.

[13] E. M. McClure, C. L. Bose, A. Garces et al., "Global network for women's and children's health research: a system for lowresource areas to determine probable causes of stillbirth, neonatal, and maternal death," Maternal Health, Neonatology and Perinatology, vol. 1, no. 1, 2015.

[14] A. G. Rouse, K. M. Gil, and K. Davis, "Diagnosis of bacterial vaginosis in the pregnant patient in an acute care setting," Archives of Gynecology and Obstetrics, vol. 279, no. 4, pp. 545549, 2009.

[15] R. M. Brotman, M. A. Klebanoff, T. R. Nansel et al., "Bacterial vaginosis assessed by gram stain and diminished colonization resistance to incident gonococcal, chlamydial, and trichomonal genital infection," Journal of Infectious Diseases, vol. 202, no. 12, pp. 1907-1915, 2010.

[16] X. Zhou, C. J. Brown, Z. Abdo et al., "Differences in the composition of vaginal microbial communities found in healthy Caucasian and black women," The ISME Journal, vol. 1, no. 2, pp. 121-133, 2007.

[17] K. C. Anukam, E. O. Osazuwa, I. Ahonkhai, and G. Reid, "Lactobacillus vaginal microbiota of women attending a reproductive health care service in Benin City, Nigeria," Sexually Transmitted Diseases, vol. 33, no. 1, pp. 59-62, 2006.

[18] P. J. Meis, M. Klebanoff, E. Thom et al., "Prevention of recurrent preterm delivery by 17 alpha-hydroxyprogesterone caproate," The New England Journal of Medicine, vol. 348, no. 24, pp. 23792385, 2003.

[19] W. W. Andrews, J. C. Hauth, and R. L. Goldenberg, "Infection and preterm birth," American Journal of Perinatology, vol. 17, no. 7, pp. 357-366, 2000.
[20] G. G. Donders, K. Van Calsteren, G. Bellen et al., "Predictive value for preterm birth of abnormal vaginal flora, bacterial vaginosis and aerobic vaginitis during the first trimester of pregnancy," BJOG: An International Journal of Obstetrics and Gynaecology, vol. 116, no. 10, pp. 1315-1324, 2009.

[21] J. C. Hauth, C. MacPherson, J. Carey et al., "Early pregnancy threshold vaginal $\mathrm{pH}$ and Gram stain scores predictive of subsequent preterm birth in asymptomatic women," American Journal of Obstetrics and Gynecology, vol. 188, no. 3, pp. 831-835, 2003. 


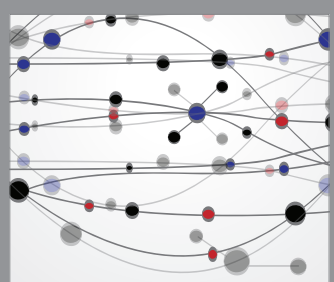

The Scientific World Journal
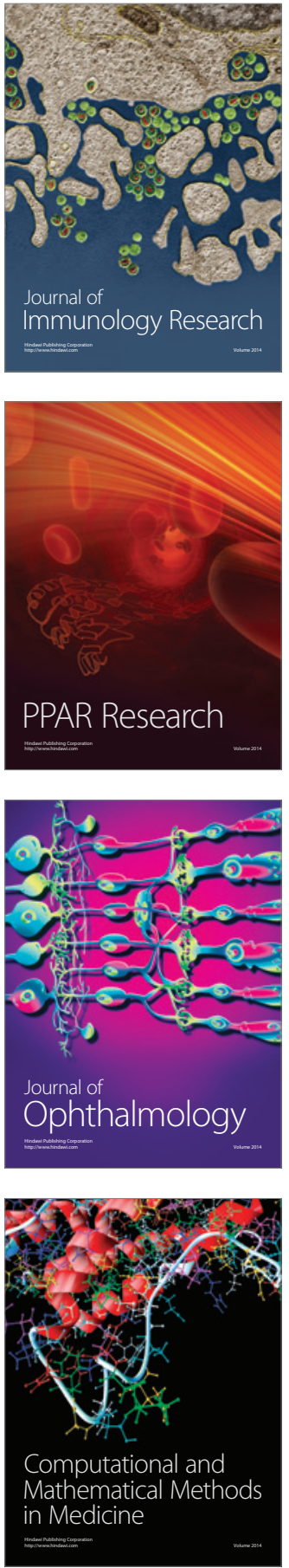

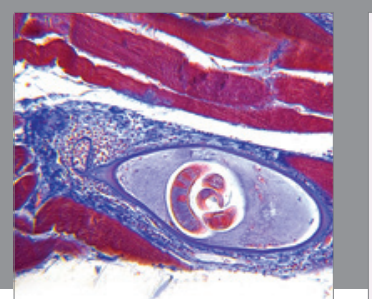

Gastroenterology Research and Practice
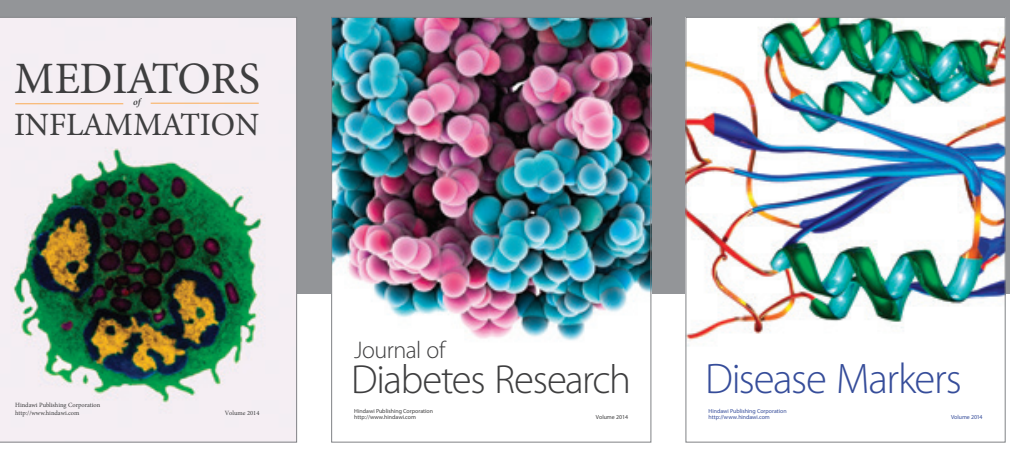

Disease Markers

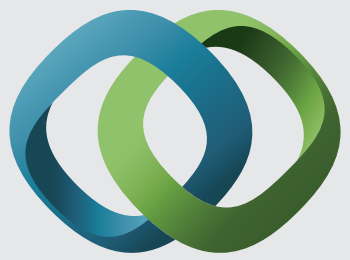

\section{Hindawi}

Submit your manuscripts at

https://www.hindawi.com
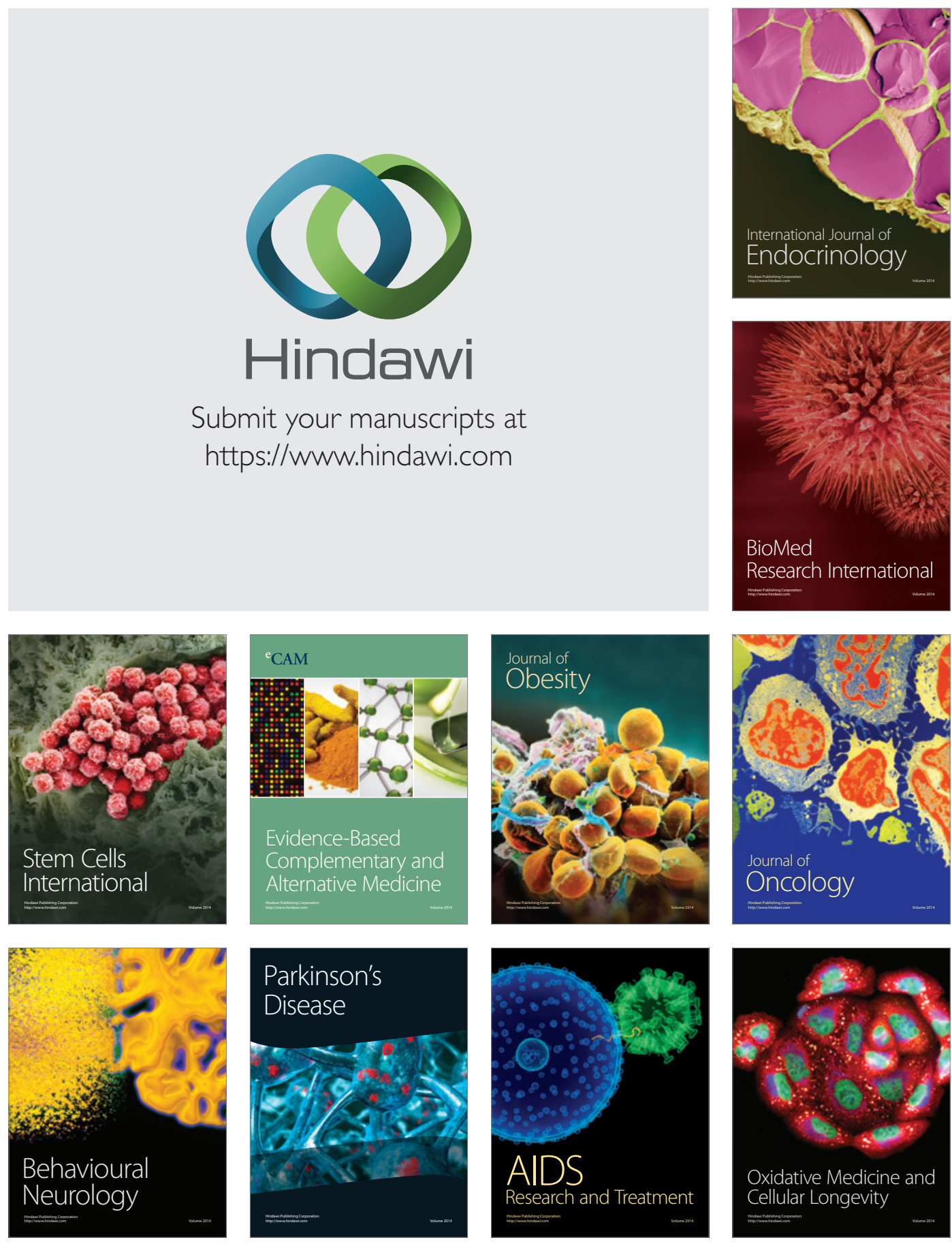